\title{
Casos de intoxicações exógenas em cães e gatos atendidos na Faculdade de Veterinária da Universidade Federal Fluminense durante o período de 2002 a 2008
}

\author{
Poisoning dogs and cats attended in the Veterinarian College of Federal Fluminense University during the \\ period of 2002 to 2008
}

\author{
Renata Jurema Medeiros $^{\mathrm{I} *}$ Fábio de Oliveira Monteiro ${ }^{\mathrm{II}}$ Gleice Castelo da Silva ${ }^{\mathrm{I}}$ \\ Amary Nascimento Júnior ${ }^{\mathrm{III}}$
}

\section{RESUMO}

Nos últimos anos, casos de intoxicação exógena de animais domésticos foram atendidos na Faculdade de Veterinária da Universidade Federal Fluminense (UFF). Sejam acidentais ou intencionais, esses casos ocorrem principalmente no ambiente doméstico e envolvem diferentes agentes tóxicos, tais como agrotóxicos, raticidas anticoagulantes, medicamentos, dentre outros. O presente trabalho objetivou realizar um estudo retrospectivo dos casos de intoxicação em cães e gatos atendidos na Faculdade de Veterinária da UFF, durante o período de março de 2002 a março de 2008. Os casos foram classificados por substância ou agente tóxico, espécie, sexo, raça, idade e ano de ocorrência. No total, foram

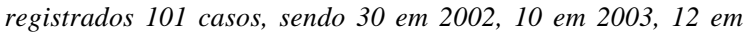
2004, três em 2005, nove em 2006 e nove casos em 2007. A maioria dos casos ocorreu no ano de 2002, tendo como principal causa a intoxicação medicamentosa, seguida da intoxicação por agrotóxicos comercializados ilegalmente como raticidas, principalmente carbamato, sendo também observado um grande número de casos em que não foi identificada a substância envolvida.

Palavras-chave cães, gatos, intoxicação exógena.

\section{ABSTRACT}

In recent years cases of domestic animals exogenous poisoned were treated at the Veterinarian College of The Federal Fluminense University (UFF). Accidentally or intentionally, they occurred mainly at home and involved different toxic agents, such as pesticides, raticides anticoagulants, and medicines, among others. This study is about a retrospective study of cases of poisoning in dogs and cats treated at the UFF veterinary college the period of March 2002 to March 2008. The cases were classified by substance or toxic agent, species, sex, breed, age and year occurrence. Totally, 101 cases were registered, with 30 in 2002, 10 in 2003, 12 in 2004, 3 in 2005, nine 9 in 2006 and 9 cases in 2007. Most cases occurred in 2002, and the principal cause was drug intoxication, followed by poisoning raticide, especially cabamate and it was also observed a large number of cases where the substance involved couldn't be identified.

Key words: dog, cat, exogenous intoxication.

\section{INTRODUÇÃO}

A cada ano, são atendidos vários casos de intoxicação exógena de animais domésticos nas clínicas e nos hospitais veterinários brasileiros. Sejam eles acidentais ou intencionais, ocorrem principalmente no ambiente doméstico e envolvem diferentes agentes tóxicos, tais como agrotóxicos de uso agrícola, agrotóxicos de uso doméstico, raticidas ou medicamentos.

Uma das principais causas de intoxicação pode ser considerada a desinformação da população quanto ao uso adequado dessas substâncias no ambiente doméstico, muitas vezes administradas ou utilizadas sem orientação ou acompanhamento de profissional qualificado, aumentando o risco de intoxicações (JONES, 1990; KENNEL et al., 1996).

IDepartamento de Zootecnia e Desenvolvimento Agro-Sócio-Ambiental Sustentável, Faculdade de Veterinária, Universidade Federal Fluminense (UFF). Rua Vital Brazil Filho, Santa Rosa, 24230-340, Niterói, RJ, Brasil. E-mail : renataa@vm.uff.br.*Autor para correspondência.

IIServiço de Anestesiologia Veterinária, Hospital Veterinário Universitário Firmino Mársico Filho (HUVET), Faculdade de Veterinária, UFF, Niterói, RJ, Brasil.

IIIDepartamento de Patologia e Clínica Veterinária e Direção do HUVET, Faculdade de Veterinária, UFF, Niterói, RJ, Brasil. 
No Brasil, dados recentes do Sistema Nacional de Informações Tóxico-Farmacológicas (SINITOX) mostram que, no ano de 2005, foram registrados pelo Centro de Informação e Assistência Toxicológica do Rio de Janeiro (CIT/RJ) 2410 casos de intoxicação humana e 113 casos de intoxicação animal e 1.577 casos de intoxicação humana e 54 casos de intoxicação animal pelo Centro de Informação e Assistência Toxicológica de Niterói (CCI/RJ - Niterói) (FIOCRUZ, 2008). Entretanto, acredita-se que esse número possa ser muito maior, uma vez que muitos casos não são notificados e nem chegam a um diagnóstico correto. Esses números referem-se, portanto, somente aos casos atendidos nos Centro de Informação e Assistência Toxicológica.

A Faculdade de Veterinária da Universidade Federal Fluminense (UFF) presta serviço à comunidade realizando o atendimento médico veterinário em suas instalações. Até o ano de 2006, o atendimento aos animais ocorreu na Policlínica Veterinária (PV). Em 2007, com a inauguração do Hospital Veterinário Universitário Professor Firmino Mársico Filho (HUVET), foi possível ampliar a quantidade de pacientes atendidos diariamente e principalmente melhorar a qualidade do atendimento e dos serviços prestados à comunidade. A antiga PV possuía uma frequência média de 20 atendimentos por dia, tornandose maior após a inauguração do HUVET, passando para uma média de 30 casos/dia, cerca de 7200 casos/ano.

Uma parcela desses atendimentos destinase ao tratamento de intoxicações exógenas envolvendo animais de companhia, que, de modo geral, têm alta representação em rotinas ambulatoriais e hospitalares na Medicina Veterinária. Frente a essa realidade e à escassez de dados sobre o perfil das intoxicações exógenas em animais, observa-se a necessidade da realização de estudos envolvendo a casuística de intoxicações em animais, contribuindo para o desenvolvimento do atendimento, do tratamento e das medidas de prevenção e controle mais eficazes, para alertar a população sobre um risco iminente de exposição a diversas substâncias, bem como para melhorar as estatísticas nacionais (ALMEIDA, 2005).

O presente trabalho teve como objetivo realizar um estudo retrospectivo para a elaboração do perfil dos casos de intoxicação exógena em cães e gatos atendidos na Faculdade de Veterinária da UFF, durante o período de março de 2002 a março de 2008, com a finalidade de aprimorar o atendimento clínico, uma vez que a rapidez e escolha correta do protocolo de atendimento são fundamentais para o sucesso no tratamento.

\section{MATERIAL E MÉTODOS}

Foram levantadas as informações obtidas nas fichas de atendimento clínico de animais atendidos na Policlínica Veterinária da UFF e no HUVET, no período de março de 2002 a março de 2008. Em seguida, foram selecionadas as fichas dos casos de intoxicação exógena confirmados e não confirmados, devido à impossibilidade de identificação do agente tóxico pelo proprietário. Os casos de intoxicação foram confirmados pela identificação do agente tóxico pelo proprietário e por meio da observação sintomatologia manifestada pelo animal no momento do atendimento clínico.

Após análise, as fichas de atendimento foram classificadas por substância ou agente tóxico, espécie, raça, sexo, idade, procedência do animal, sintomatologia e ano de ocorrência. Os agentes tóxicos responsáveis pelas intoxicações foram classificados em sete grupos: medicamentos (uso humano e veterinário), praguicidas agropecuários, rodenticidas (cumarínicos), domissanitários, plantas tóxicas, acidente ofídico e substância desconhecida ou casos não confirmados.

\section{RESULTADOS E DISCUSSÃO}

O número total de atendimentos no período analisado foi de 15.568 casos, correspondente a 1.632 casos em 2002, $1.560 \mathrm{em} \mathrm{2003,} 1.904 \mathrm{em} 2004,2.312 \mathrm{em}$ 2005, 2.040 em 2006, 4.352 casos em 2007 e 1.768 casos em 2008. Deste total, foram registrados 101 casos de intoxicação.

Em relação às substâncias e ao sexo dos animais, foram 22 casos de intoxicação por agrotóxicos; 22 casos de intoxicação por medicamentos de uso humano e veterinário (21,8\%), com predomínio de fêmeas maiores de 12 meses e sem raça definida; 14 casos (13,9\%) por agrotóxicos, sem predomínio de sexo, sendo Poodle a raça mais acometida em animais com menos de 12 meses; oito casos de intoxicação com raticidas cumarínicos (7,9\%), sem predomínio de raça e com predomínio de animais maiores de 12 meses; seis casos (5,9\%) por domissanitários; seis casos $(5,9 \%)$ por plantas tóxicas; três casos (3,0\%) por acidentes ofídicos; e 34 casos (33,7\%) não confirmados ou por substâncias desconhecidas. Os dados relatados podem ser visualizados nas tabelas 1 e 2

Os cães corresponderam a 86,1\% (87 casos) e os gatos apenas 13,9\% (14 casos) do total de casos. Dados semelhantes foram observados por HORNFELDT \& MURPHY (1997) em estudo realizado nos Estados Unidos, no período de 1993 a 1994, em 
Casos de intoxicações exógenas em cães e gatos atendidos na Faculdade de Veterinária da Universidade Federal Fluminense... 2107

Tabela 1 - Classificação das substâncias envolvidas nos casos de intoxicação em cães atendidos na Faculdade de Veterinária da Universidade Federal Fluminense, durante o período de 2002 a 2008.

\begin{tabular}{|c|c|c|c|c|c|c|c|c|}
\hline \multirow{2}{*}{ Substâncias } & \multicolumn{8}{|c|}{ 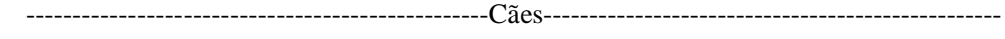 } \\
\hline & 2002 & 2003 & 2004 & 2005 & 2006 & 2007 & 2008 & $\mathrm{~N}$ \\
\hline Agrotóxicos & & & & & & & & 23 \\
\hline Carbamatos & 3 & 2 & 2 & & 1 & 5 & & \\
\hline Organofosforados & & & 1 & & & & & \\
\hline Piretrinas/Piretróides & 2 & 1 & 1 & & 1 & 3 & & \\
\hline Neocide ${ }^{\circledR}$ (organofosforado + piretrina) & 1 & & & & & & & \\
\hline Med. Veterinários & & & & & & & & 8 \\
\hline Amitraz & 2 & & 1 & & & & & \\
\hline Ivermectina & & 1 & & & 2 & 2 & & \\
\hline Med. Humanos & & & & & & & & 8 \\
\hline Acido salicílico + enxofre & 1 & & & & & & & \\
\hline Antidepressivos & 1 & & & & & & & \\
\hline Perrmetrina & 1 & & & & & & & \\
\hline Sulfato de Vincristina & & 1 & & & & & & \\
\hline Acetaminofen+diclofenaco & & & 1 & & & & & \\
\hline Clorazepan & & & & 1 & & & & \\
\hline Diclofenaco & & & & & & & 1 & \\
\hline Cumarínicos & 1 & 2 & & 1 & 1 & 1 & 2 & 8 \\
\hline Domissanitários & & & & & & & & 6 \\
\hline Desinfetantes & 1 & & 1 & & & 1 & & \\
\hline Hipoclorito & & & & & & 2 & & \\
\hline Sabão em pó & & & & & & 1 & & \\
\hline Plantas Tóxicas & & & & & & & & 6 \\
\hline Dieffenbachia picta & & & & & & 3 & & \\
\hline Ricinus comunis & & & 1 & & & & 1 & \\
\hline Sansevieria zeylanica & 1 & & & & & & & \\
\hline Acidente Ofídico & & & & & & & & 3 \\
\hline Bothrops sp. & 1 & 1 & & & & & & \\
\hline Serpente não identificada & & & & & & 1 & & \\
\hline Subs. Desconhecidas & 4 & 2 & 1 & 1 & & 6 & 1 & 15 \\
\hline Casos não confirmados & 4 & & 1 & & 1 & 6 & & 12 \\
\hline Total de casos por ano & 23 & 10 & 10 & 3 & 6 & 31 & 5 & 88 \\
\hline
\end{tabular}

que $82,8 \%$ dos casos de intoxicação ocorreram em cães e 13,6\% em gatos. Em 2004, FORRESTER observou, nos dados do Centro de Intoxicação do Texas, que $87 \%$ das intoxicações ocorreram em cães e 11\% em gatos. No Brasil, em estudo prévio similar, XAVIER et al. (2002) observaram que os cães foram responsáveis por $81,2 \%$ e os gatos por $18,8 \%$ dos casos de intoxicação analisados. Em estudo realizado na Áustria, no período de 1999 a 2004, foram identificados em 123 animais positivos para intoxicação por agrotóxicos. Deste total, 47,2\% foram cães e 34,1\% gatos (WANG et al., 2007).
A diferença entre os números de intoxicações entre cães e gatos pode estar relacionada à característica dos felinos de serem mais seletivos que os caninos com sua alimentação, além de serem capazes de recusar obstinadamente qualquer alimento que apresente um odor que não lhes agrade. Esse número menor de casos é um fator positivo para a espécie, uma vez que os gatos apresentam peculiaridades metabólicas que podem favorecer um quadro de intoxicação quando comparados a outras espécies animais. A primeira característica está relacionada à deficiência relativa na conjugação de substâncias, 
Tabela 2 - Classificação das substâncias envolvidas nos casos de intoxicação em gatos atendidos na Faculdade de Veterinária da Universidade Federal Fluminense, durante o período de 2002 a 2008.

\begin{tabular}{|c|c|c|c|c|c|c|}
\hline \multirow{2}{*}{ Substâncias } & \multirow[b]{2}{*}{2002} & \multirow[b]{2}{*}{2004} & \multirow[b]{2}{*}{2006} & \multirow[b]{2}{*}{2007} & \multirow[b]{2}{*}{2008} & \multirow[b]{2}{*}{$\mathrm{N}$} \\
\hline & & & & & & \\
\hline \multicolumn{7}{|l|}{ Med. Veterinários } \\
\hline Monosulfureto de tetraetiltiuran & & 1 & & & & 1 \\
\hline Azul de Metileno & & & & 1 & & 1 \\
\hline \multicolumn{7}{|l|}{ Med. Humanos } \\
\hline Fenazopiridina & 1 & & & & & 1 \\
\hline Cetoconazol & 1 & & & & & \\
\hline Acido Acetil Salicílico & & 1 & & & 1 & 2 \\
\hline Subs. Desconhecidas & & & 1 & 1 & 2 & 4 \\
\hline Casos não confirmados & & & & 1 & 2 & 3 \\
\hline Total de casos por ano & 2 & 2 & 1 & 3 & 4 & 12 \\
\hline
\end{tabular}

especificamente na fase 2 da biotransformação (fase de conjugação das substâncias ou dos metabólitos da fase 1), em que o gato apresenta deficiência relativa no processo de conjugação com o ácido glicurônico em virtude de concentrações extremamente baixas ou deficiência de algumas enzimas da família glicoroniltransferases. Portanto, muitas substâncias metabolizadas por essa via apresentam uma meia-vida prolongada nesses animais. A segunda característica está relacionada ao fato de os felinos apresentarem, na composição de seus eritrócitos, de oito a 20 grupos sulfidril reativos, enquanto em humanos são somente dois e nos cães quatro, assim há maior susceptibilidade dessa espécie a sofrer lesões oxidativas na presença de um agente oxidante (SOUZA, 2003).

Os casos de intoxicação medicamentosa correspondem ao maior percentual dos casos analisados. Esse perfil foi similar ao descrito por XAVIER et al. (2002 e 2008) e atribui-se ao uso inadequado dos medicamentos em animais, sem respeitar as individualidades de cada espécies e principalmente as diferenças de metabolização das substâncias. Esse fato está relacionado, na maioria das vezes, a desinformações dos proprietários que não procuram a orientação de um médico veterinário e realizam, assim como a automedicação, a administração arbitrária de medicamentos aos animais.

As intoxicações por medicamento de uso veterinário estão relacionadas aos casos de incorreta administração e comercialização desses produtos devido à prescrição de medicamentos diversas vezes orientada pelos atendentes dos estabelecimentos comerciais (pets shops) e lojas de produtos agropecuários ou, muitas vezes, pelo próprio proprietário sem a consulta a um médico veterinário.
Como exemplo, podem-se citar os casos de indicação de "mata bicheira” (azul de metileno) para felinos e ivermectina em dose inadequada para ação carrapaticida em cães da raça Collie e Pastor Shetland, que possuem sensibilidade a essas substâncias, podendo ocasionar a morte (XAVIER et al., 2008).

Em relação aos agrotóxicos, dados do SINITOX demonstram que em 2005, no Brasil, esses agentes foram responsáveis por 12.788 (15,5\%) casos de intoxicação humanas e 565 (40,9\%) intoxicações animais. Destes, os agrotóxicos de uso doméstico foram responsáveis por 2.590 casos de intoxicação humana e 205 casos de intoxicação animal (FIOCRUZ, 2008).

No presente estudo, destacaram-se as intoxicações por carbamato, substância conhecida vulgarmente como "chumbinho" e vendida ilegalmente a partir do tráfico do aldicarb. Esse dado vai ao encontro aos estudos realizados em 2007 por WANG et al. e XAVIER et al., em que o carbamato foi indicado como a principal causa de intoxicação aguda, acidentais ou não. Segundo os autores, esse fato está relacionado à alta toxicidade de alguns compostos, principalmente o aldicarb (Temik ${ }^{\circledR}$ ), um agente anticolinesterásico (carbamato) comercializado de forma clandestina e usado ilegalmente como raticida doméstico, à facilidade de aquisição de produtos registrados para uso agrícola contendo essas substâncias e ao fato de a fiscalização da comercialização de agrotóxicos ser ainda ineficiente.

As intoxicações por raticidas cumarínicos aparecem como a segunda maior causa responsável por 7,92\% dos casos de intoxicação analisados, seguidas pelos agrotóxicos organosfosforados, que foi responsável por apenas um caso de intoxicação. Esses dados são similares aos observados no estudo realizado por WANG et al. (2007), no qual os raticidas

Ciência Rural, v.39, n.7, out, 2009. 
anticoagulantes foram igualmente a segunda maior causa das intoxicações, seguidos dos agrotóxicos organosfosforados responsáveis por 18,9\% e 5,1\% dos casos confirmados, respectivamente.

Em relação aos inseticidas piretrinas e piretróides, foram observados somente casos de intoxicação em cães (7,1\%). Entretanto, em estudo realizado por SUTTON et al. (2007), foi observado que, em 1306 casos de intoxicação por permetrina, substância do grupo das piretrinas e piretróides, 80,9\% das intoxicações ocorreram em gatos, e os autores atribuem esse fato às características anteriormente comentadas e relacionadas à dificuldade dos felinos em detoxificar algumas substâncias.

Ainda em relação aos agrotóxicos, no presente estudo, foi possível observar um caso de intoxicação em cão por Neocid ${ }^{\circledR}$, um inseticida em pó indicado para combater pulgas e formigas no ambiente. Segundo o próprio fabricante, este não deve ser aplicado diretamente sobre pessoas ou animais. Esse dado aponta a desinformação da população quanto ao uso adequado de substâncias perigosas no ambiente doméstico. Estas muitas vezes são administradas ou utilizadas sem orientação, de forma incorreta, expondo homens e animais ao risco de intoxicações graves.

No grupo do domissanitários, foram observados apenas seis casos de intoxicação em animais que possuíam idade inferior a oito meses, assim como nos acidentes por plantas tóxicas comigoninguém-pode (Dieffenbachia picta), mamona (Ricinus comunis) e espada de São Jorge (Sansevieria zeylanica), nos quais a idade média dos animais também foi de cerca de cinco meses.

Os acidentes ofídicos somaram um total de apenas três casos. Dois casos foram relacionados a serpentes do gênero Bothrops, e o terceiro não foi possível de ser identificada a serpente envolvida. Pela sintomatologia, suspeitou-se de outro acidente botrópico com a realização de tratamento correspondente.

A ocorrência de intoxicações por agentes tóxicos não identificados foi pronunciada. Nesses casos, foi observada a dificuldade dos proprietários em fornecer informações precisas sobre a ocorrência para o médico veterinário. Um histórico incompleto associado à desinformação da população e à falta de recursos laboratoriais para os testes toxicológicos adequados dificultam a identificação da substância envolvida, ficando evidente que somente pela sintomatologia nem sempre é possível identificar o agente causador da intoxicação ou desenvolver um tratamento adequado. Muitas substâncias podem provocar sintomas semelhantes, porém o tratamento indicado para cada quadro clínico é muito diferente. Esse fato é ainda mais importante se as diferenças metabólicas e fisiológicas entre as espécies animais forem confrontadas.

\section{CONCLUSÕES}

A maioria dos casos de intoxicação ocorreu no ano de 2002, sendo os medicamentos a principal causa de intoxicação identificada, seguidos por agrotóxicos utilizados ilegalmente como raticida, principalmente o "chumbinho" (carbamato). Foi observado também um grande número de casos em que não foi possível identificar a substância envolvida, o que é um fator complicador para o tratamento.

Diante dessas informações, é de fundamental importância a capacitação dos profissionais da área em relação às medidas de primeiros socorros e ao tratamento adequado para os diferentes casos de intoxicação. Entretanto, a prevenção ainda é a melhor maneira de reduzir a incidência de intoxicações em animais de companhia, sendo necessário o desenvolvimento de educação continuada para conscientização da população em relação à utilização adequada de produtos tóxicos em potencial.

\section{REFERÊNCIAS}

ALMEIDA, C.G. Intoxicação em cães e gatos: estudo retrospectivo dos casos atendidos em um Centro Veterinário Privado durante o período de 1996 a 2004. In: CONGRESSO BRASILEIRO DE TOXICOLOGIA, 14., 2005, Recife. Anais... Recife: Revista Brasileira de Toxicologia, 2005. p. 296.

FIOCRUZ. Sistema Nacional de Informações Tóxicofarmacológicas (SINITOX). Capturado em 08. Jul. 2008. Online. Disponível em: <http://www.fiocruz.br/sinitox/2005/ tab4_brasil.pdf $>$.

HORNFELDT, C.S.; MURPHY, M.J. Poisonings of animals: the 1993-1994 report of the American Association of Poison Control Centers. Vet Hum Toxicol, v.39, n.6, p.361-365, 1997.

JONES, R.D. Xylene/Amitraz: a phamacologic review en profile. Vet Hum Toxicol, v.32, n.5, p.446-44. 1990.

KENNEL, O. et al. Four cases of amitraz poisoning in humans. Vet Hum Toxicol, v.38, n.1, p.28-30, 1996.

SOUZA, H.J.M. Coletâneas em medicina e cirurgia felina. Rio de Janeiro: L.F. Livros de Veterinária, 2003. V..1, 477p.

FORRESTER M.B. Pattern of stingray injuries reported to Texas poison centers from 1998 to 2004. Hum Exp Toxicol, n.24, p.639-642, 2005. 
SUTTON, N. et al. Clinical effects and outcome of feline permethrin spot-on poisonings reported to the Veterinary Poisons Information Service (VPIS), London. Journal of Feline Medicine and Surgery, n.9, p. 335- 339, 2007. Disponível em: <http://www.sciencedirect.com/ science?_ob=ArticleURL\&_udi=B6WJC-4P5RW36$1 \&$ _user $=686357 \&$ \&_doc $=1 \&$ \& $\mathrm{fmt}=\&$ \&orig $=$ search $\&$ _sort $=\mathrm{d}$ $\&$ view $=$ c\&_acct $=$ C $000037518 \&$ \&version $=1 \&$ \&_urlVers ion=0\&_userid=686357\&md5=5e1b6a7a9836c2cb9c348c82e5edeaf6 $>$. Acesso em: 20 abr. 2009. doi:10.1016

XAVIER, F.G. et al. Common causes of poisoning in dogs and cats in a Brazilian Veterinary Teaching Hospital from 1998 to 2000. Vet Human Toxicol, v.44, n.1, p.115-116, 2002.
XAVIER, F. G. et al. Toxicologia do praguicida aldicarb ("chumbinho"): aspectos gerais, clínicos e terapêuticos em cães e gatos. Ciênc Rural, v.37, n.4, p.1206-1211, 2007. Disponível em: <http://www.scielo.br/scielo.php?script=sci_arttext\&pid=S0103$84782007000400051>$. Acesso em: 20 nov. 2008. doi: 10.1590/ S0103-84782007000400051.

XAVIER, F.G. et al. Toxicologia dos medicamentos. In: SPINOSA, H.S. et al. Toxicologia aplicada à medicina veterinária. São Paulo: Manole, 2008. Cap.6, p.117-189.

WANG, Y. et al. Pesticide poisoning in domestic animals and livestock in Austria: A 6 years retrospective study. Forensic Sci Int, v.169, n.2-3, p.157-160, 2007. 